\title{
HIGHLIGHTS
}

PUBLIC HEALTH

\section{Ozone pollution-a link with cardiac and cerebral ischemic events?}

Urban pollution is a major cause of respiratory health problems, although the specific effect of ozone $\left(\mathrm{O}_{3}\right)$ exposure on cardiovascular disease is not wellknown. Jean-Bernard Henrotin and co-workers have found that short-term $\mathrm{O}_{3}$ exposure can trigger recurrent ischemic cerebrovascular events (ICVE) and myocardial infarction (MI), especially in individuals with severe vascular risk factors.

As part of the population-based Dijon Vascular Project (DIVA), the investigators obtained data on ICVE and MI in Dijon, France, between 2001 and 2007 from the Stroke Registry of Dijon and the Registry of MI of Dijon and Côte d'Or, respectively. Overall, 1,574 ICVE and 913 MI were included in the analysis, and data for vascular risk factors, such as history of hypertension, diabetes mellitus, and hypercholesterolemia, were incorporated in the study. Air pollution data for the same time period were acquired from the Dijon Environmental Monitoring Centre and included measurements of $\mathrm{O}_{3}$, sulfur dioxide, nitrogen dioxide, and carbon monoxide levels.

To calculate the effect of $\mathrm{O}_{3}$ exposure on ICVE and MI, the researchers assessed the relationship between the levels of $\mathrm{O}_{3}$ on the day of each cerebral and cadiac event and for the 4 days leading up to the events. $\mathrm{O}_{3}$ exposure was significantly associated with recurrent ICVE with a 3-day lag (odds ratio [OR] 1.115, 95\% CI 1.027-1.209), although a similar association with recurrent MI was not significant. Furthermore, both recurrent and incident ICVE were significantly associated with $\mathrm{O}_{3}$ exposure at a lag time of 1 day in patients with diabetes mellitus (OR 1.138, 95\% CI 1.027-1.260). By contrast, similar associations between $\mathrm{O}_{3}$ exposure and recurrent and incident $\mathrm{MI}$ were only found in patients with a history of hypercholesterolemia (OR 1.111, 95\% CI 1.020-1.211).

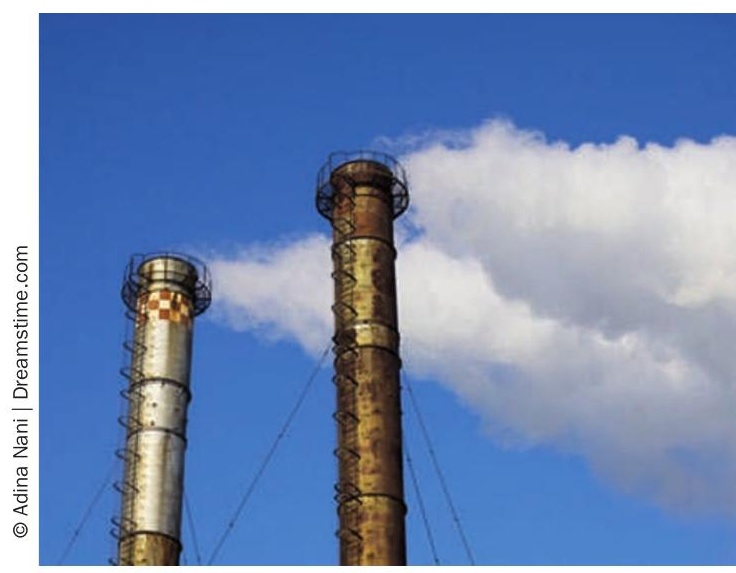

These findings suggest that exposure to $\mathrm{O}_{3}$ might increase the risk of ischemic events, particularly in individuals with vascular risk factors. The authors propose that an inflammatory reaction induced by $\mathrm{O}_{3}$ might be responsible for the cardiac and cerebral ischemic events.

\section{Rowan Higgs}

